\title{
Study of Time-Dependent Properties of Thermoplastics
}

\author{
V. A. Kolupaev ${ }^{1, a}$, A. Bolchoun ${ }^{1}$ and H. Altenbach ${ }^{2}$ \\ 1 German Institute for Polymers (DKI), Schlossgartenstrasse 6, D-64289 Darmstadt \\ 2 Martin-Luther-Universität Halle-Wittenberg, Lehrstuhl für Technische Mechanik, D-06099 Halle
}

\section{Summary}

Simple tests carried out with a common tension/compression testing machine are used to obtain timedependent properties of non-reinforced thermoplastics. These tests include ramp loadings as well as relaxation and creep tests. Two materials (PBT Celanex 2002-2 and POM Hostaform C9021, Ticona $\mathrm{GmbH}, \mathrm{Kelsterbach}$ ) were taken for the experiments. The experiments show that an adequate description of the long-term material properties can be obtained from the short-time tests, namely from tests with constant traverse speed $\dot{L}$.

Below a model for the time-dependent mechanical behavior is presented and fitted to the obtained measured data. For the evaluation of the fitting quality long-term tests are used. Especially creep and relaxation tests with "jumps", i.e. rapid change of loading, are important for this purpose.

\section{Experiments}

The following experiments were carried out: ramp loading with constant stress rate $\dot{\sigma}=$ const and with constant traverse speed $\dot{L}=$ const, standard creep and relaxation tests as well as creep and relaxation tests with rapid change of loading ("jumps"). The ramp loading tests are short-time ones, the remaining tests are long-time. The deformations occurring during the tests are captured on a suitable element of the specimen's surface using the optical measuring system Vic-2D, Limess Messtechnik \& Software $\mathrm{GmbH}$, Pforzheim.

The ramp loading tests are simple and with the setting $\dot{L}=$ const relatively stable. It is shown that these tests are sufficient to obtain the parameters of the BAILEY-NorTON function

$$
\dot{\varepsilon}=e \sigma^{n} t^{g}, \quad e>0, \quad n>1, \quad-1<g \leq 0,
$$

the elastic modulus $E$ as well as the parameter $\alpha$, which describes the material response to "jumps". The mentioned properties are required to fit the model to the measured data.

If the material properties should be obtained from the long-time experiments, so the creep test only is not sufficient, and relaxation test is required (Fig. 1). The tests with "jumps" are essential in order to capture the material properties. A test with three "jumps" is used in order to control the quality of fitting. Since the strain is measured in both the longitudinal and the transverse directions, it is possible to compute the PoIsson's ratio [2]. This can be used as an additional verification for the model.

\footnotetext{
a e-mail: VKolupaev@dki.tu-darmstadt.de
} 

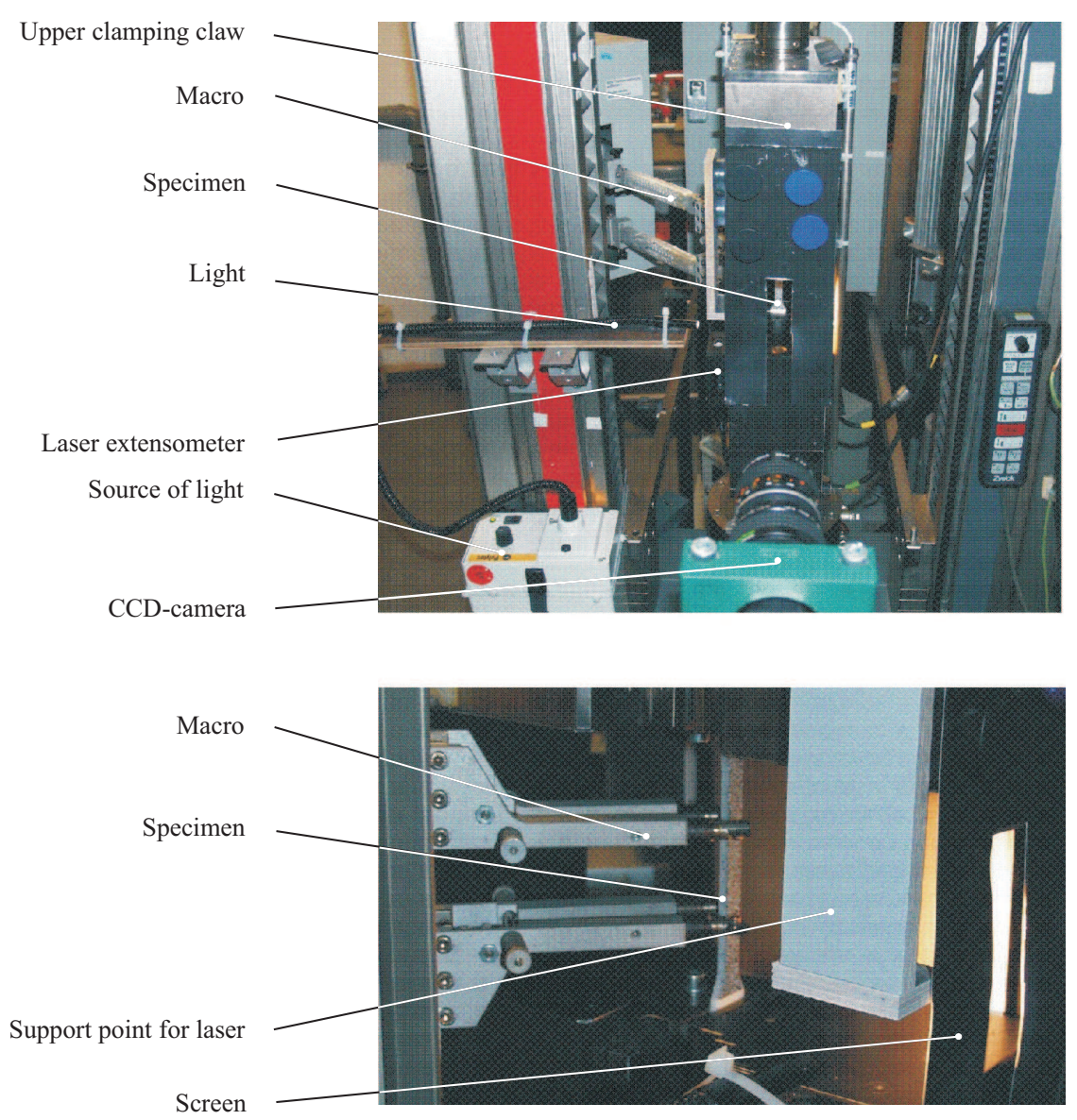

Fig. 1. Testing machine Zwick Z020 with a CCD-camera. Setup for the relaxation test

\section{Model and Fitting to the Experimental Results}

For the modeling of the time-dependent behavior a model of type

$$
\dot{\varepsilon}=\sigma^{n} \frac{\mathrm{d} \lambda(t)}{\mathrm{d} t}+\alpha n \frac{\mathrm{d} \sigma}{\mathrm{d} t} \sigma^{n-1} \lambda(t)+\frac{1}{E} \frac{\mathrm{d} \sigma}{\mathrm{d} t} \quad \text { with } \quad \alpha \in[0,1]
$$

can be used [2]. This model contains the total strain $(\alpha=1)$ as well as the time hardening $(\alpha=0)$ theories [5]. The simplest expression for the function $\lambda(t)$ is $\lambda(t)=e t^{g}$, and the common BAILEYNorton function is obtained. The parameter $\alpha$ determines the response of the model to rapid changes of loading ("jumps").

The main goal of the fitting is: to fit the model to the observed material behavior and to determine an "optimal" set of experiments, that means to identify the experiments, which yield all necessary information about the material behavior as quick as possible.

The fitting of the model is carried out in few steps. At first, the elastic properties are determined. The best results are achieved using the test with constant traverse speed $\dot{L}=0,001 \mathrm{~mm} / \mathrm{s}$. The Emodulus is computed according to the ISO-527 norm:

$$
E=\frac{\sigma_{2}-\sigma_{1}}{\varepsilon_{2}-\varepsilon_{1}}
$$

with $\varepsilon_{1}=0.0005, \varepsilon_{2}=0.0025$ and $\sigma_{1}, \sigma_{2}$ are the respective stresses. 
Second step can be carried out in two different ways. An optimization routine can be used to determine the parameters of the BaILEY-NoRTon function and then the parameter $\alpha$. Alternatively the parameters of the BAILEY-NoRTON function and $\alpha$ can be computed simultaneously.

The fitting routine requires the computation of stress as well as strain rates $\dot{\varepsilon}$ and $\dot{\sigma}$. These derivatives are computed as symmetric differential quotients. No filter is applied, since the measured data are sufficiently smooth. The results of fitting are presented in fig. 2, 3, 4, 5 .

For the second step it is required to normalize the time into the interval $[0,1]$. If the measurements from different tests are used for fitting, then the normalization must be uniform. More precisely there are time series $t_{i}^{j}, i=1, . ., n_{j}$. Put

$$
n_{\max }:=\max _{j} t_{n_{j}}
$$

and the normalized time series $s_{i}^{j}, i=1, . ., n_{j}$ are obtained as follows:

$$
s_{i}^{j}=\frac{t_{i}^{j}}{n_{\max }} .
$$

For further computations with the model it is required to keep the value $n_{\max }$ and use it as a normalizing coefficient for the time series. It is important, if the time derivatives have to be computed. For an accurate fitting it is important to have either tests with "jumps" or to have ramp loading tests for different loading speeds.

The results of fitting are presented in fig. 2, 3, 4, 5. Here the model is used to compute stress from the measured strain. Since the model can be used in both directions (to compute strain from the available strain data and vice versa) it is a natural thing to do in order to verify the model.

\section{References}

1. V.A. Kolupaev, 3D-Creep Behaviour of Parts Made of Non-Reinforced Thermoplastics (in German) (Martin-Luther Universität Halle-Wittenberg, Papierflieger Verlag, Clausthal- Zellerfeld, 2006)

2. V. A. Kolupaev, A. Bolchoun, 12. Tagung Deformations- und Bruchverhalten von Kunststoffen 24-26.06.2009, Book of Abstracts ed. by W. Grellmann, Merseburg (2009), pp. 340-344

3. V. A. Kolupaev, A. Bolchoun, H. Altenbach, Konstruktion 5, (2009) pp. $59-66$

4. V.A. Kolupaev, H. Altenbach, A. Bolchoun, 1st International Conference on Material Modelling, September 15th-17th 2009, Dortmund (2009), p. 176

5. R. K. Penny, D. L. Marriott, Design for creep (McGraw-Hill, London, 1971)

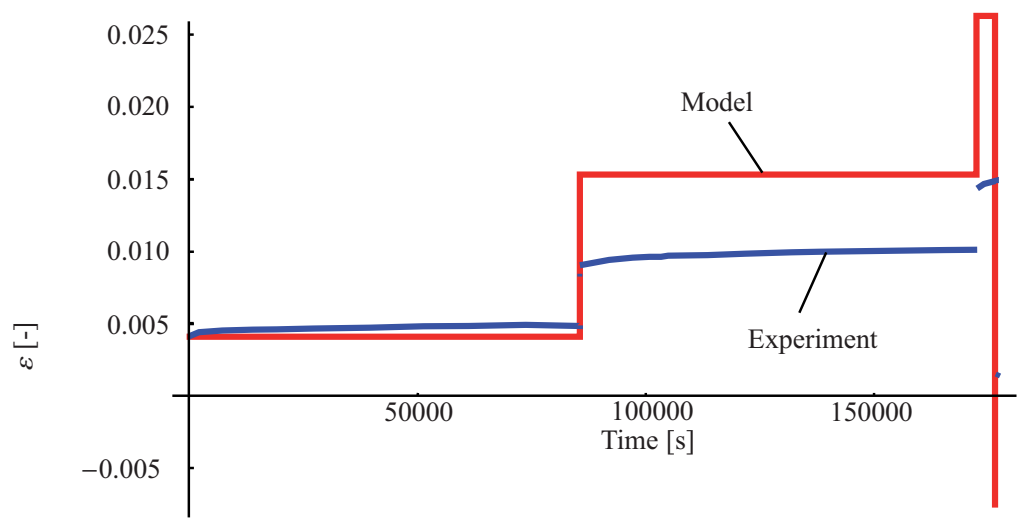

Fig. 2. 3-step creep. Time-strain-diagram for PBT 2002-2. The parameters of the model were fitted using the tests with constant traverse speed: $E=2643, n=1.15, g=0.00018, e=0.00028, \alpha=0.45$. 


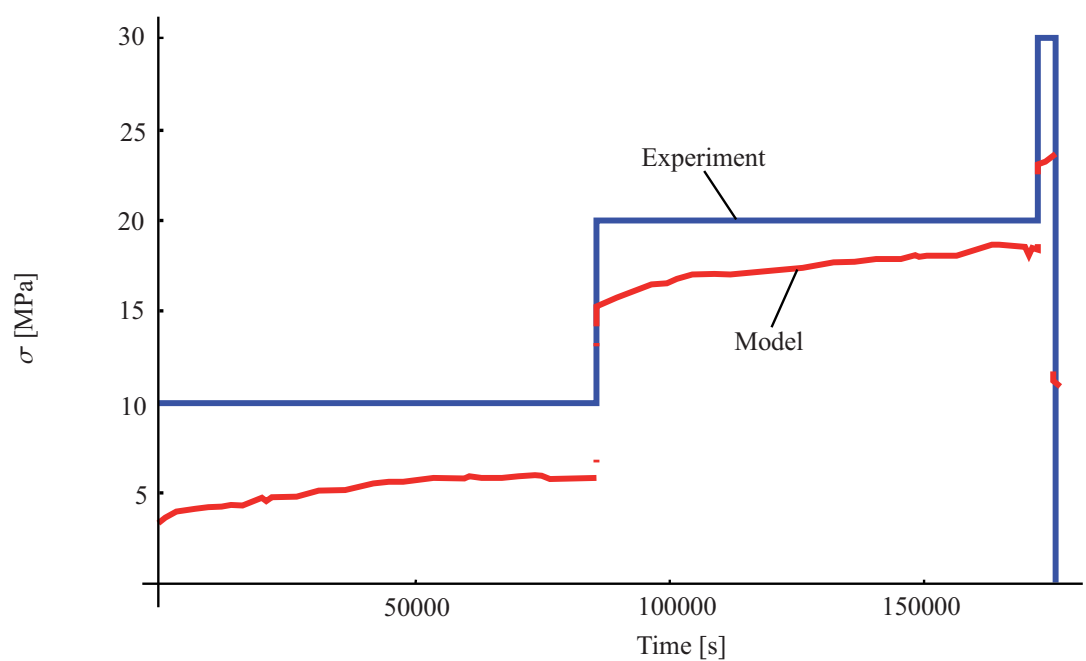

Fig. 3. 3-step creep. Time-stress diagram for PBT 2002-2. The parameters of the model were fitted using the tests with constant traverse speed: $E=2643, n=1.15, g=0.00018, e=0.00028, \alpha=0.45$.

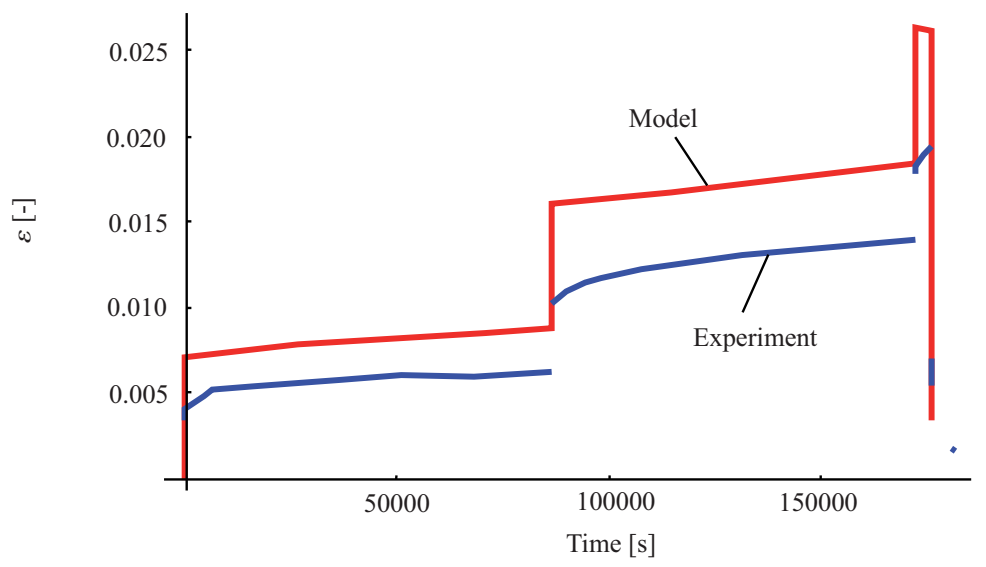

Fig. 4. 3-step-creep. Time-strain diagram for POM C9021. The parameters of the model were fitted using the tests with constant traverse speed: $E=2831, n=1.19, g=0.67, e=0.000011, \alpha=0.56$.

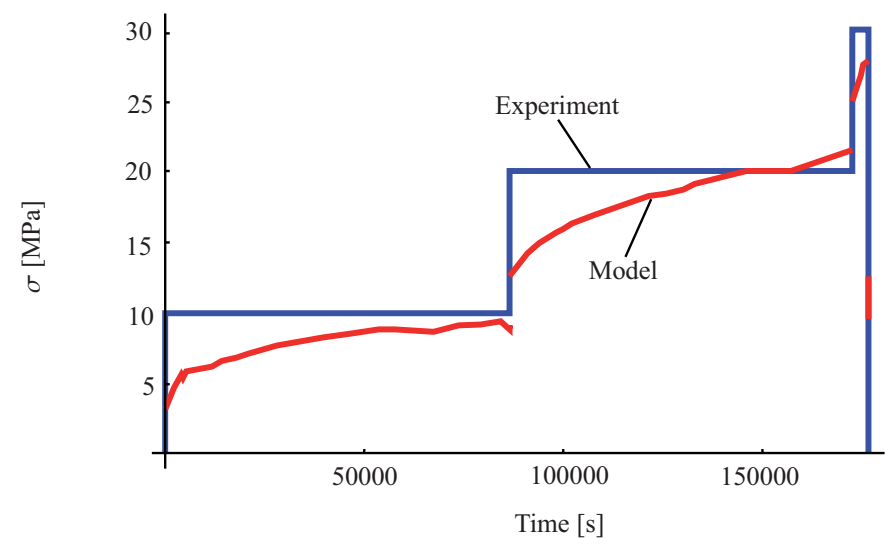

Fig. 5. 3-step creep. Time-stress diagram for POM C9021.The parameters of the model were fitted using the tests with constant traverse speed: $E=2831, n=1.19, g=0.67, e=0.000011, \alpha=0.56$. 